\title{
A Comparative Analysis Of Once-Daily And Twice- Daily Formulation Of Tacrolimus In De Novo Kidney Transplant Recipients
}

Murat Ferhat Ferhatoglu ( $\nabla$ ferhatferhatoglu@yahoo.co.uk )

Okan Universitesi https://orcid.org/0000-0003-1520-7517

Abdulcabbar Kartal

Okan Universitesi

Ali Ilker Filiz

Okan Universitesi

Gursel Yildiz

Okan Universitesi

Alp Gurkan

Okan Universitesi

Research

Keywords: Kidney transplantation, immunosuppression, tacrolimus, prolonged-release tacrolimus.

Posted Date: July 28th, 2020

DOl: https://doi.org/10.21203/rs.3.rs-45454/v1

License: (9) This work is licensed under a Creative Commons Attribution 4.0 International License. Read Full License

Version of Record: A version of this preprint was published at SiSli Etfal Hastanesi Tip Bulteni / The Medical Bulletin of Sisli Hospital on January 1st, 2020. See the published version at https://doi.org/10.14744/SEMB.2020.71235. 


\section{Abstract}

Objectives: Once-daily formulation of tacrolimus has begun to find a place in daily clinical practice as similar efficacy and safety profile as the twice-daily formulation according to clinical trials. However, few numbers of un-sponsored, non-trial studies are issued centering on its usage in clinical practice. We compared once-daily and twice-daily formulation of tacrolimus in terms of the efficiency and effects on graft function in de novo kidney transplant patients.

Methods: Twenty once-daily (TAC-OD) and twenty twice-daily (TAC-BID) tacrolimus administrated de novo kidney recipients who had received initial immunosuppressive therapy according to protocols at our institution $(0.2 \mathrm{mg} / \mathrm{kg}$ of tacrolimus combined with 1000 milligrams of steroid taper plus $720 \mathrm{mg}$ of mycophenolate and with $2,5 \mathrm{mg} / \mathrm{kg}$ human immune globulin) assessed in terms of demographics, drug doses and blood concentration, and graft function.

Results: The mean tacrolimus blood concentration measurements were higher in the TAC-OD group in the first sixty days after transplantation, and the TAC-OD group showed more blood concentration overshoots in the first 30 days of the treatment. The initial drug dose was significantly higher in the TAC-OD group compared to the TAC-BID group ( $p=0.04)$. There was no meaningful difference among groups according to graft function (creatinine measurements) ( $p>0.05)$.

Conclusion: Between de novo kidney recipients, the new TAC-OD formulation presents a similar short-term efficacy profile as TAC-BID. However, a higher daily dosage of TAC-OD is needed to achieve similar blood concentrations in the early postoperative period.

\section{Introduction}

Tacrolimus is a milestone immunosuppressive drug in the field of organ transplantation, as proved by the outcomes of numerous researches [1-3]. Tacrolimus has presented a notable decrease in the frequency and severity of acute allograft rejection episodes in solid organ (kidney, liver, and heart) transplants with enhanced graft survival in the long term [1, 4].

The absence of new immunosuppressant medications in solid organ transplantation has inspired the scientists to move forward by altering known immunosuppressive therapies for advancing long term results. A prolonged-release new formulation (FK506E) was recently delivered (TAC OD, Advagraf, Astellas PharmaUS, Inc., Deerfield, III, United States), which allows for once-daily treatment of tacrolimus while keeping a safe and efficient blood concentration similar to twice-daily dosing. However, the data on this issue is scarce in the literature $[5,6]$.

Many authorities believe, non-adherence to immunosuppressant medication is the most significant problem on preventable graft loss after solid organ transplantation [7, 8]. Once-daily dosing of tacrolimus instead of twice-daily dosing may enhance adherence to medication and improve long-term outcomes. Nevertheless, to date, an insufficient number of studies have analyzed the difference in blood- 
concentration and dosing profile of once-daily and twice-daily dosing tacrolimus at de novo kidney transplant patients $[3,7,9]$.

The once-daily dosing of tacrolimus initiated in 2019 in our clinic, and we questioned in which recipient groups (once-daily tacrolimus vs. twice-daily tacrolimus) it was simpler to reach and sustain the target/ therapeutic blood concentration of tacrolimus $(8-12 \mathrm{ng} / \mathrm{ml})$ in the early postoperative period. We also compared once-daily and twice-daily formulation of tacrolimus in terms of the efficiency and effects on graft function in de novo kidney transplant patients.

\section{Materials And Methods}

Inclusion criteria: We reviewed the medicinal data of de novo kidney recipients who received initial immunosuppressant therapy with once-daily tacrolimus (TAC-OD), conducted between January 2019 and February 2020 at our institution. We compared them with a group of 20 kidney transplants, who were randomly selected, using equivalent immunosuppression with twice-daily tacrolimus (TAC-BID), and performed between January 2019 and December 2019.

Preoperative management: The liver and kidney chemistry, complete blood count, coagulation profile, Tand $B$ - cell cross matches, ABO blood group, serum for tissue typing tests, serology for CMV, EBV, VZV, toxoplasma, hepatitis B and C, HIV, pregnancy test $f$ appropriate, urine culture (unless anuric), chest x-ray, electrocardiogram, pelvic duplex ultrasonography for the evaluation of iliac vessels were completed.

Immunosuppressant therapy protocol: According to protocols at our institution, $0.2 \mathrm{mg} / \mathrm{kg}$ of tacrolimus, a calcineurin inhibitor, was started as an induction agent the night before the surgery. Furthermore, 500 milligrams of steroid taper, methylprednisolone, with $720 \mathrm{mg}$ of mycophenolate mofetil was administrated the operation morning. Lastly, $500 \mathrm{mg}$ additional dose of steroid taper, methylprednisone, with $2,5 \mathrm{mg} / \mathrm{kg}$ human immune globulin was also applied during operation.

Slow graft function: Serum creatinine $>3 \mathrm{mg} / \mathrm{dL}$ on postoperative day five but not requiring dialysis [10].

Warm ischemia time: The time in seconds from clamping of the renal artery until obtaining clear efflux from the renal vein on the bench [11].

Cold ischemia time: Time interval between cold solution perfusion and revascularization, was incomplete [12].

Compared variables: Demographics, Human leukocyte antigen mismatch, co-morbid conditions, serum creatinine levels, warm and cold ischemia times were analyzed. Also, serum creatinine levels, immunosuppressant therapy at 7th, 30th, 60th, and 180th days after operation were evaluated. Tacrolimus doses and blood concentrations in both groups were analyzed.

Statistical Analysis: We practiced the Number Cruncher Statistical System-2007® (USA) computer application for statistical evaluation of the study data. We used descriptive statistical techniques (mean, 
standard deviation, median, first quadrant, third quadrant, frequency, percentage, minimum, maximum) for the evaluation of data. Fisher's Exact test was applied to analyze the qualitative data. Statistical significance was taken as $p<0.05$.

\section{Results}

Overall, 40 kidney recipients enrolled in the study protocol. No significant differences between recipients using TAC-OD and TAC-BID were observed according to demographics and clinical data demonstrated in Table 1. There was one acute rejection observed in either TAC-OD and TAC-BID groups. The mean tacrolimus blood concentration measurements were higher in the TAC-OD group in the first sixty days after transplantation; five patients' tacrolimus blood concentration was higher than target concentration; however, the level curves in both groups were similar between day- 60 and day- 180 after transplantation (Fig. 1). The initial tacrolimus blood concentration was higher in the TAC-OD group (Fig. 1), and the TACOD group showed more blood concentration overshoots in the first 30 days of the treatment (Fig. 2). Additionally, more patients were under target concentration in the first month of the treatment in the TACBID group (Fig. 3), but two recipients in either group were under target concentration after six-months of therapy. The blood concentration curves were smoother (less fluctuating) in recipients receiving twicedaily tacrolimus (Fig. 3). 
Table 1

Demographics and clinical data of kidney transplant recipients on once-daily and twice-daily tacrolimus

Once-daily tacrolimus ( $\mathrm{n}$

= 20)

Recipient age (years), Mean \pm SD (min-max)

Recipient gender, $\mathrm{n}(\%)$

Recipient BMI* $(\mathrm{kg} / \mathrm{m} 2)$, Mean \pm SD (min$\max )$

Donor age (years), Mean \pm SD (min-max)

Donor gender, $\mathrm{n}(\%)$

Donor type, n (\%)

Comorbidities, n (\%)

Diabetes mellitus

$9(45)$

7 (35)

Hypertension

Coronary artery disease

Chronic kidney disease etiology, n (\%)

Diabetes mellitus

Hypertension

Kidney stone disease

Polycystic kidney disease

Focal segmental glomerulosclerosis

Amiloidosis

Membranoproliferative glomerulonephritis

Unknown

HLA* type mismatch, Mean

Type A

1.12

0.88

Type B

0.54

0.5

Type DR-B1
11 (55)

$2(10)$

$10(50)$

5 (25)

7 (35)

3 (15)

1 (5)

1 (5)

$0(0)$

1 (5)
$6(30)$
4 (20)

(30)

wice-daily tacrolimus ( $n$

$42.8 \pm 14.4(26-64)$

10 (50) male/ 10 (50) female

$27,5 \pm 4.9(16.6-32.1)$

$48.5 \pm 14(30-65)$

1 (55) male/ 9 (45) emale

8 (90) living/ 2 (10) deceased 


\begin{tabular}{|lll|}
\hline & $\begin{array}{l}\text { Once-daily tacrolimus }(\mathbf{n} \\
\mathbf{2} 20)\end{array}$ & $\begin{array}{l}\text { Twice-daily tacrolimus }(\mathbf{n} \\
\mathbf{=} 20)\end{array}$ \\
\hline Type DQ-B1 & 0.56 & 0.56 \\
\hline PRA* grade, $\mathbf{n}(\%)$ & $20(100)$ & $1(5)$ \\
\hline $0-50$ & $0(0)$ & $1(5)$ \\
\hline$>50$ & $2,09 \pm 0.45(1.5-3)$ & $1.87 \pm 0.55(1.5-3)$ \\
\hline $\begin{array}{l}\text { Warm ischemia time (minutes), Mean } \pm \text { SD } \\
\text { (min-max) }\end{array}$ & $58 \pm 10.99(41-76)$ & $61.54 \pm 44.7(42-77)$ \\
\hline $\begin{array}{l}\text { Cold ischemia time (minutes), Mean } \pm \text { SD } \\
\text { (min-max) }\end{array}$ & $1(5)$ & $1(5)$ \\
\hline Acute rejection, $n$ (\%) & $0(0)$ & $0(0)$ \\
\hline Graft loss, $n$ (\%) & $1(5)$ & $2(10)$ \\
\hline CMV infection, $n(\%)$ & & \\
\hline *BMI: Body mass index, HLA: Human leukocyte antigen, PRA: Panel reactive antibody \\
\hline
\end{tabular}

The initial drug dose was significantly higher in the TAC-OD group compared to the TAC-BID group $(p=$ 0.04). The dose adjustments needed for optimal blood concentration were higher in the TAC-OD group, but the difference was non-significant $(p>0.05)$ (Fig. 4).

There was no meaningful difference among groups according to graft function (creatinine measurements) ( $p>0.05)$. One graft from the TAC-OD group and two grafts from the TAC-BID group showed slow graft function, but these three grafts were obtained from deceased donors (Table 2). Also, none of the recipients suffered severe liver dysfunction. 
Table 2

Creatinine measurements and immunsupressive theraphy (except tacrolimus) of the recipients once-daily and twice-daily tacrolimus

\begin{tabular}{|lll|}
\hline & $\begin{array}{l}\text { Once-daily tacrolimus } \\
(\mathbf{n}=\mathbf{2 0})\end{array}$ & $\begin{array}{l}\text { Twice-daily tacrolimus } \\
(\mathbf{n}=\mathbf{2 0})\end{array}$ \\
\hline Creatinine (mg/dl), Mean \pm SD (min-max) & & \\
\hline Day 7 & $1.34 \pm 0.37(0.69-5.19)$ & $1.58 \pm 0.69(0.7-4.12)$ \\
\hline Day 30 & $1.14 \pm 0.25(0.6-3.25)$ & $1.2 \pm 0.39(0.81-2-32)$ \\
\hline Day 60 & $1.23 \pm 0.21(0.8-2)$ & $1.39 \pm 0.58(0.74-1.9)$ \\
\hline Day 180 & $1.21 \pm 0.37(0.77-2.1)$ & $1.34 \pm 0.56(0.84-2.26)$ \\
\hline Steroid dose (gr/kg), Mean \pm SD (min-max) & & \\
\hline Day 7 & $0.26 \pm 0.04(0.18-0.48)$ & $0.31 \pm 0.08(0.20-0.44)$ \\
\hline Day 30 & $0.15 \pm 0.05(0.09-0.18)$ & $0.16 \pm 0.05(0.11-0.26)$ \\
\hline Day 60 & $0.10 \pm 0.04(0.07-0.16)$ & $0.10 \pm 0.05(0.07-0.09)$ \\
\hline Day 180 & $0.05 \pm 0.04(0.03-0.06)$ & $0.06 \pm 0.04(0.02-0.10)$ \\
\hline $\begin{array}{l}\text { Mycophenolic acid dose (gr/kg), Mean } \pm \text { SD } \\
\text { (min-max) }\end{array}$ & & \\
\hline Day 7 & $1.89 \pm 0.35(1.3-3.5)$ & $2.04 \pm 0.63(1.5-3.6)$ \\
\hline Day 30 & $1.44 \pm 0.62(1.1-2.6)$ & $1.88 \pm 0.69(1.6-2.7)$ \\
\hline Day 60 & $1 \pm 0.34(0.9-1.6)$ & $1.21 \pm 0.46(1.1-1.9)$ \\
\hline Day 180 & $0.81 \pm 0.15(0.62-1.34)$ & $1.07 \pm 0.16(0.8-1.4)$ \\
\hline
\end{tabular}

\section{Discussion}

The presented study showed that recipients using de novo TAC-OD needed higher drug doses to achieve the target blood concentration even if the baseline demographics of the recipients were identical. However, the sixth-month graft function in patients using TAC-OD and TAC-BID was similar.

Several studies revealed that TAC-OD required higher daily doses up to six months after transplantation than those on TAC-BID $[2,13,14]$. Kitada et al. showed that recipients on TAC-OD therapy have lower tacrolimus blood concentration and necessitate to be administered higher doses than those receiving TAC-BID in the early posttransplant phase [15]. Our outcomes on daily dosage were consistent with previous studies; recipients having TAC-OD immunosuppressive therapy needed higher doses than those receiving TAC-BID. Tacrolimus is principally absorbed from the intestines. The inactive components inserted into the capsule covering the active drug form create disparity among once-daily and twice-daily forms $[14,15]$. This prolonged-release period may be linked with problems in optimizing the blood 
concentration in the target concentration through the initial post-transplant period. Several determinants (polymorphisms of the CYP3A enzyme [16, 17], daily-diet [18], and intestinal motility [19] have been associated with the absorption of tacrolimus.

Romina et al. observed that de novo TAC-OD administrated kidney recipients had lower blood concentration than TAC-BID using patients. They also observed that TAC-OD using recipients and recipients who changed medication from TAC-BID to TAC-OD three months after transplantation had an excellent functioning graft and did not occur acute rejection [2]. However, Morales et al. revealed that there was a significant reduction in tacrolimus blood concentration after switching to TAC-OD. They required to increase the daily dosage of tacrolimus to keep the blood concentration on the target level [20]. We did not notice any trouble in the graft capacity of TAC-OD, or TAC-BID received patients after the six months in our study, and TAC-OD was effective in de novo as TAC-BID.

In the presented study, the creatinine levels were insignificantly higher in recipients who were using TACBID. This finding was also noticed in patients who were switched from TAC-BID to TAC-OD on long-term observation. The advance in graft function looked to be independent of fluctuations in tacrolimus blood concentration $[15,21]$.

\section{Limitations of the study}

This study should be analyzed in light of some limitations. Retrospective, single-institution conducted nature, and the limited number of individuals is the foremost obstacle of our research. Multicenter conducted with larger sample size investigations are needed to ratify our findings. Secondly, several investigations have implied that non-adherence is a notable reason for graft failure. Non-adherence to medication is an essential determinant that should be discussed $[6,7,15]$. We attempted to ascertain the incidence of non-adherence by utilizing a self-administered questionnaire. Although all of the TAC-OD and TAC-BID recipients were de novo cases, adherence to medication was sufficient in both groups. Nevertheless, we decided that the results were unreliable in our study groups. We think that medication adherence characteristics can be best evaluated in patients who have switched from TAC-BID to TAC-OD. Also, considering all individuals included in the present study had caucasian ethnicity, presented conclusions may not be generalizable to kidney recipients with different ethnicities.

\section{Conclusion}

De novo TAC-OD therapy after kidney transplantation is reliable, ensuring equal stability in drug blood concentrations compared to the TAC-BID form, and did not have any negative influence on graft function. However, recipients using de novo TAC-OD after kidney transplantation need higher daily medication doses than TAC-BID, but this dosage difference tends towards non-significance over time.

\section{Declarations}


Ethical approval statement: All procedures performed in studies in- volving human participants were in accordance with the ethical standards of the institutional and/or national research committee and with the 1964 Helsinki Declaration and its later amendments or comparable ethical standards.

Consent for publication: Written and oral consent were obtained from all participians

Availability of data and materials: Not applicable.

Competing interests: The authors declared no conflict of interest.

Funding: There is no funding related to this article

Authors' contributions: MFF, AK, GY collected the information, reviewed the literature, and wrote the manuscript. AIF critically reviewed the manuscript, and AG approved the final form. All authors read and approved the final manuscript.

Acknowledgements: None

\section{Authors information:}

${ }^{1}$ Istanbul Okan University, Faculty of Medicine, Department of General Surgery, Istanbul, Turkey

${ }^{2}$ Istanbul Okan University, Faculty of Medicine, Department of Nephrology, Istanbul, Turkey

\section{References}

1. Banas B, Krämer BK, Krüger B, Kamar N, Undre N. Long-Term Kidney Transplant Outcomes: Role of Prolonged-Release Tacrolimus. Transplant Proc.2020; 52: 102- Doi:

10.1016/j.transproceed.2019.11.003.

2. Danguilan RA, Lamban A, Pamugas GEP. Pilot Study Comparing the Efficacy, Safety, Convertibility, and Tacrolimus Trough Levels of Twice-Daily Tacrolimus (Prograf) to Once-Daily Tacrolimus (Advagraf) Among Standard-Risk Kidney Transplant Patients at the National Kidney and Transplant Institute. Transplant Proc.2019; 51: 2615- Doi: 10.1016/j.transproceed.2019.05.019.

3. Sukkha S, Suansanae T, lamrahong P, Wiwattanathum P. Trough Level and Tacrolimus Variability of Early Converted Once-Daily Tacrolimus: 1-Year Follow-up Study. Transplant Proc.2020; 52: 775Doi: 10.1016/j.transproceed.2019.12.039.

4. First MR. First clinical experience with the new once-dailyformulation of tacrolimus. Ther Drug Monit. 2008;30:159-66. Doi:10.1097/FTD.0b013e318167909a.

5. First MR, Fitzsimmons WE. Modified release tacrolimus. Yonsei Med J. 2004;45:1127-31.

Doi:10.3349/ymj.2004.45.6.1127. 10.1016/j.transproceed.2020.02.109

6. Kamińska D, Poznański P, Kuriata-Kordek M, et al. Conversion From a Twice-Daily to a Once-Daily Tacrolimus Formulation in Kidney Transplant Recipients. Transplant Proc. 2020; 30: 183-4. 
Doi: 10.1016/j.transproceed.2020.02.109. 10.1016/j.transproceed.2018.06.027

7. Fellström B, Holmdahl J, Sundvall N, Cockburn E, Kilany S, Wennberg L. Adherence of Renal Transplant Recipients to Once-daily, Prolonged-Release and Twice-daily, Immediate-release Tacrolimus-based Regimens in a Real-life Setting in Sweden. Transplant Proc.2018; 50: 3275- Doi: 10.1016/j.transproceed.2018.06.027.

8. Rathi M, Rajkuma V, Rao N. Conversion from tacrolimus to cyclosporine in patients with new-onset diabetes after renal transplant: An open-label randomized prospective pilot study. Transplant Proc. 2015; 47:1158- Doi: 10.1016/j.transproceed.2014.12.050.

9. Saengram W, Vadcharavivad S, Poolsup N, Chancharoenthana W. Extended release versus immediate releasetacrolimus in kidney transplant recipients: a systematic review and meta-analysis. Eur J Clin Pharmacol. 2018; 74: 1249- Doi: 10.1007/s00228-018-2512-7.

10. Liu Y,Liu H, Shen Y, Chen Y, Cheng Y. Delayed Initiation of Tacrolimus Is Safe and Effective in Renal Transplant Recipients With Delayed and Slow Graft Transplant Proc. 2018; 50: 2368-70. Doi: 0.1016/j.transproceed.2018.03.101

11. Kukla U,Cholewa H, Chronowska J. Effect of the Second Warm Ischemia Time and Its Components on Early and Long-term Kidney Graft Function. Transplant Proc. 2016; 48: 1365-9. Doi: 1016/j.transproceed.2015.11.042.

12. Davari H, Malek-Hossini S, Salahi H, et al.Sequential anastomosis of accessory renal artery to external iliac artery in the management of renal transplantation with multiple arteries. Transplant Proc. 2003; 35: 329-31. Doi: 1016/s0041-1345(02)03838-1

13. Crespo M, Mir M, Marin M,et al. De novo kidney transplant recipients need higher doses of Advagraf compared with Prograf to get therapeutic levels. Transplant Proc. 2009; 41: 2115- Doi: 10.1016/j.transproceed.2009.05.014

14. Marquet $\mathrm{P}$, Albano L, Woillard JB, et al. Comparative clinical trial of the variability factors of the exposure indices used for the drug monitoring of two tacrolimus formulations in kidney transplant recipients. Pharmacol Res. 2018; 129: 84- Doi: 10.1016/j.phrs.2017.12.005

15. Kitada H, Okabe Y, Nishiki T, et al. One-Year Follow-Up of Treatment With Once-Daily Tacrolimus in De Novo Renal Transplant. Exp Clin Transplant.2012; 10: 561- Doi: 10.6002/ect.2012.0087.

16. Satoh S, Saito M, Inoue T, et al. CYP3A5 *1 allele associated with tacrolimus trough concentrations but not subclinical acute rejection or chronic allograft nephropathy in Japanese renal transplant recipients. Eur J Clin Pharmacol.2009; 65: 473- Doi: 10.1007/s00228-008-0606-3

17. Tsuchiya N, Satoh S, Tada H, et al. Influence of CYP3A5 and MDR1 (ABCB1) polymorphisms on the pharmacokinetics of tacrolimus in renal transplant recipients. Transplantation. 2004; 78: 1182- Doi: 10.1097/01.tp.0000137789.58694.b4

18. Bekersky I, Dressler D, Mekki QA. Effect of low- and high-fat meals on tacrolimus absorption following $5 \mathrm{mg}$ single oral doses to healthy human subjects. J Clin Pharmacol. 2001; 41: 176- Doi: $10.1177 / 00912700122009999$ 
19. Bekersky I, Dressler D, Mekki Q. Effect of time of meal consumption on bioavailability of a single oral 5 mg tacrolimus dose. J Clin Pharmacol. 2001; 41: 289- Doi: 10.1177/00912700122010104

20. Morales JM, Varo E, Lázaro P. Immunosuppressant treatment adherence, barriers to adherence and quality of life in renal and liver transplant recipients in Spain. Clin Transplant. 2012; 26: 369- Doi: 10.1111/j.1399-0012.2011.01544.x

21. Kolonko A, Chudek J, Wiecek A. Improved kidney graftfunction after conversion from twice daily tacrolimus to a once dailyprolonged-release formulation. Transplant Proc 2011; 43: 2950- Doi: 10.1016/j.transproceed.2011.07.014.

\section{Figures}

12

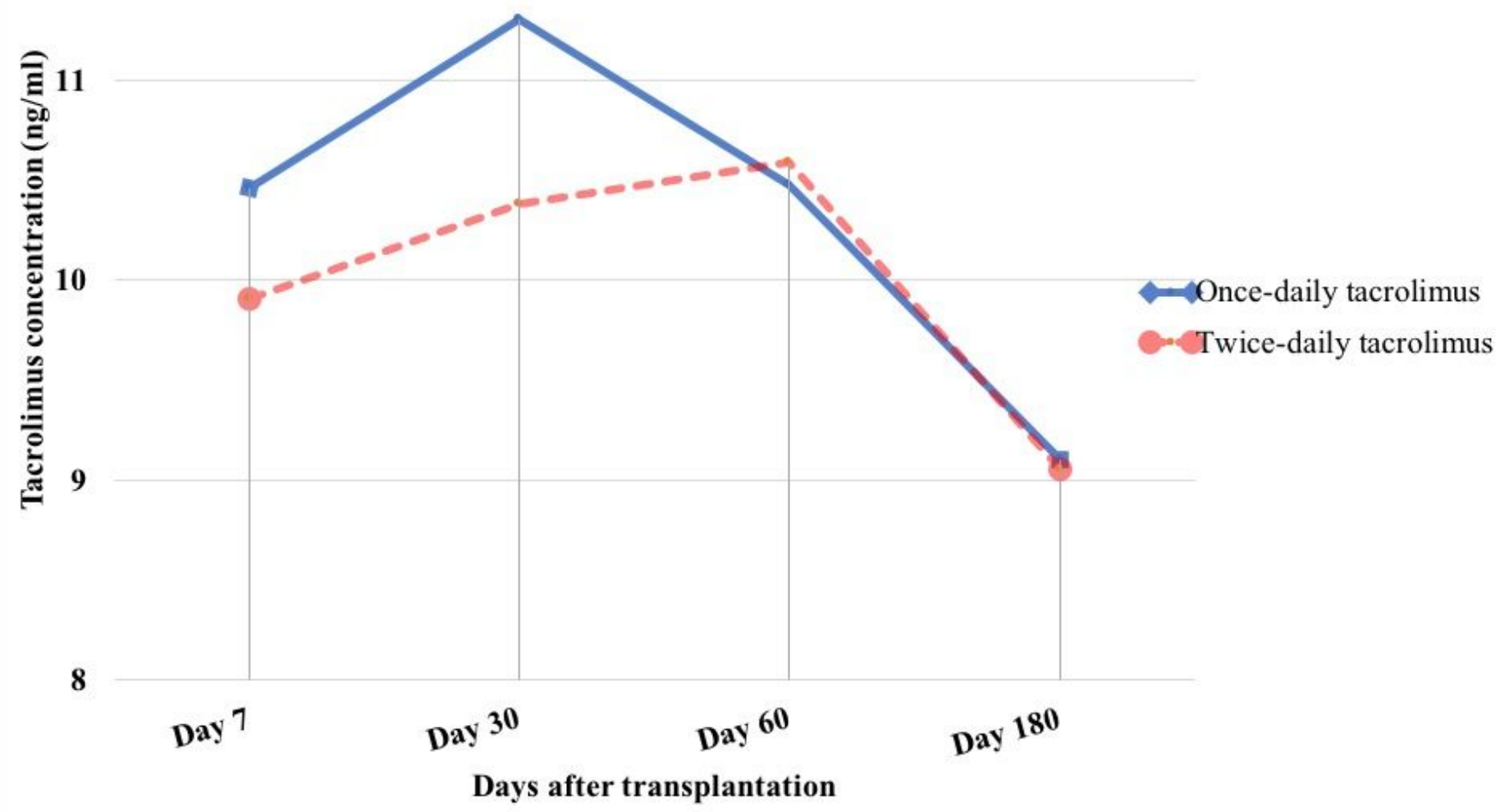

Figure 1

Tacrolimus levels among kidney recipients using once-daily and twice-daily tacrolimus 


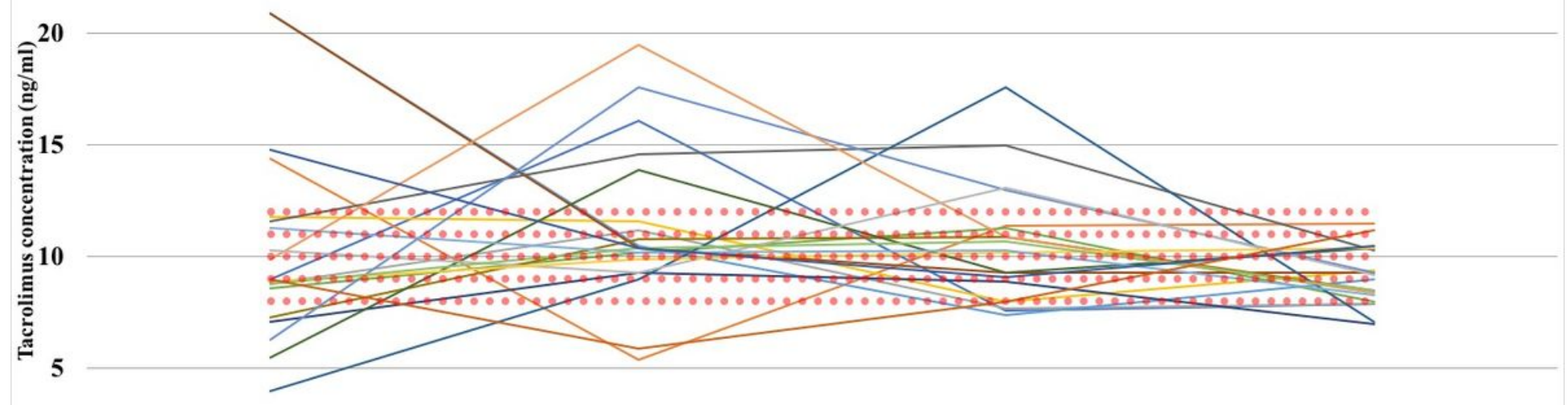

0
Day 7
Day 30
Day 60
Day 180
Days after transplantation

Figure 2

Therapeutic blood concentration monitoring of once-daily tacrolimus (Red dots indicates the expected theraupeutic range of tacrolimus level $(8-12 \mathrm{ng} / \mathrm{ml})$ in our clinic)

25

Twice-daily tacrolimus

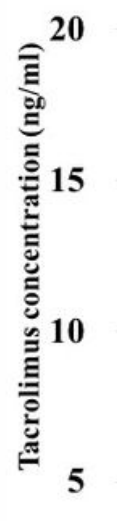

0

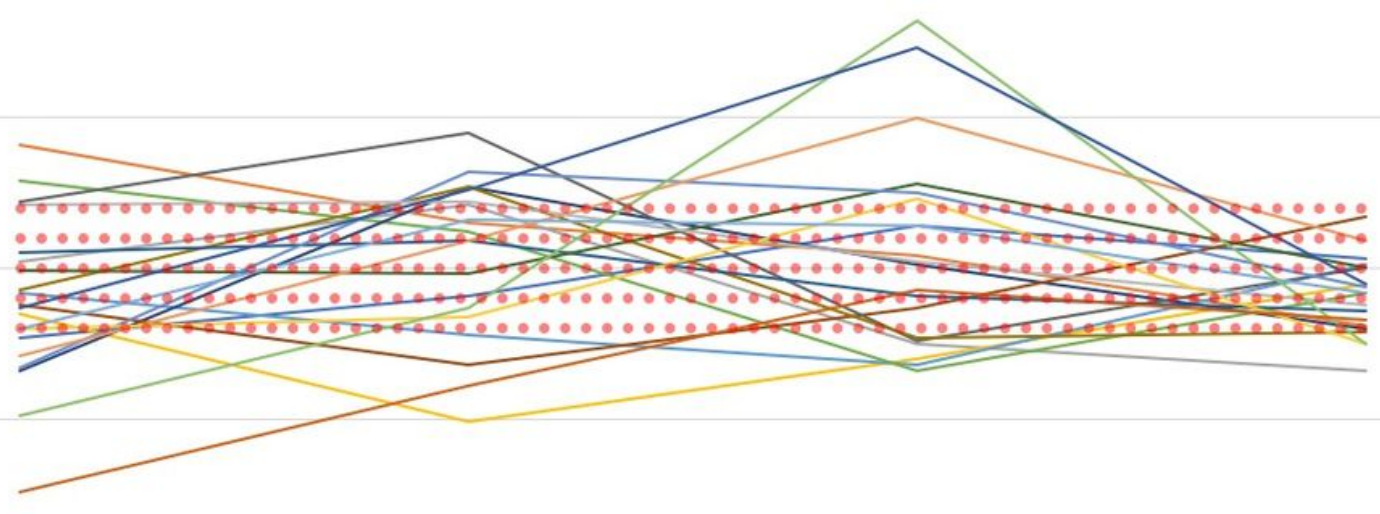

Day 7

Day 30

Day 60

Day 180

Days after transplantation

Figure 3

Therapeutic blood concentration monitoring of twice-daily tacrolimus (Red dots indicates the expected theraupeutic range of tacrolimus level $(8-12 \mathrm{ng} / \mathrm{ml})$ in our clinic) 
0,3

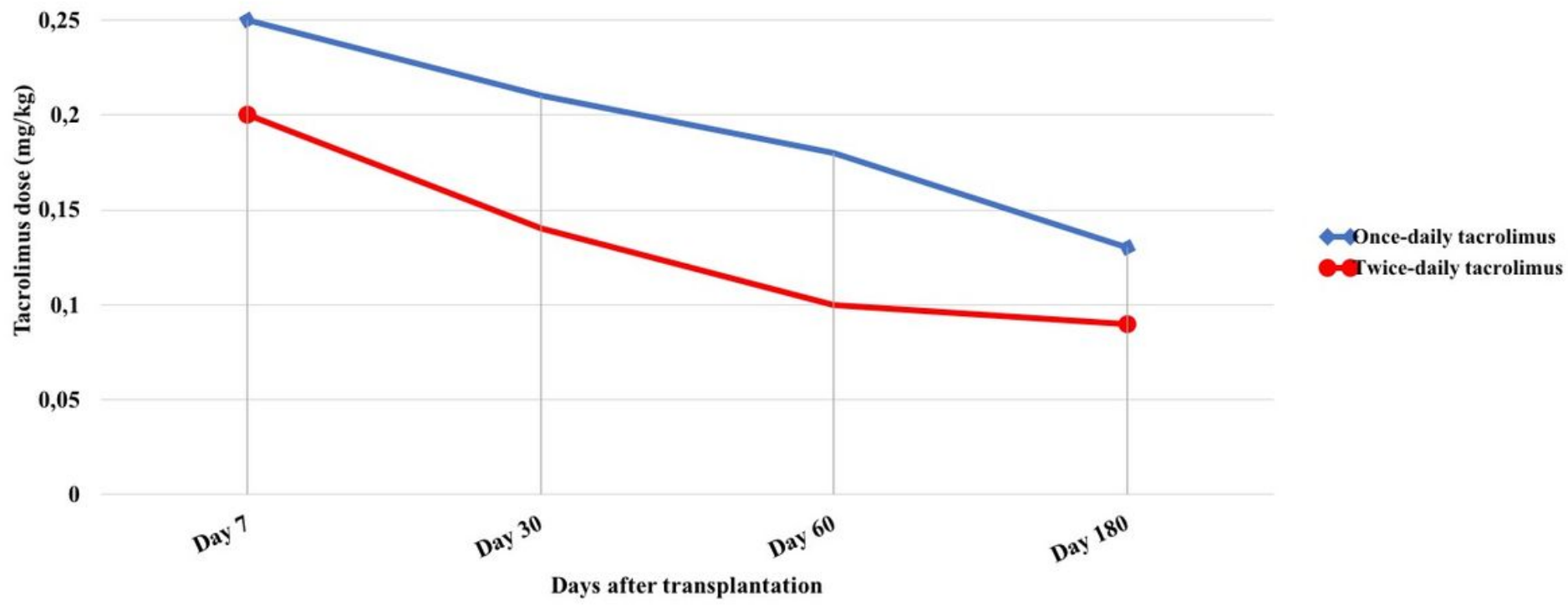

Figure 4

Tacrolimus doses among kidney recipients using once-daily and twice-daily tacrolimus 